\title{
Scientific Meeting of the Faculty of Accident and Emergency Medicine, The Royal College of Surgeons of England, London, 24-25 January, 1997
}

\author{
Ankle effusions following acute ankle \\ injury \\ N K Makvvana, P A Evans, D Finlay, W M \\ Harper \\ Trauma and Orthopaedics, Leicester Royal \\ Infirmary, Walnut Street, Leicester LE1 5WW
}

We prospectively reviewed 56 patients presenting to A\&E with an ankle effusion following injury. An ankle effusion was detected on the lateral radiograph as a tear shaped opacity displacing the normal fat adjacent to the anterior or posterior margin of the ankle joint. Joint effusions following trauma are often associated with a significant injury in the elbow and knee. An ankle effusion may be an indicator of serious injury and it has been proposed that effusions over $13 \mathrm{~mm}$ should be investigated further. We reviewed 56 patients presenting with an ankle effusion following injury prospectively. The $x$ rays were reviewed by an experienced radiologist and the size of effusion, soft tissue swelling and soft tissue index (STI) was determined. Patients were assessed clinically and functionally at 6 months.

Results-Most injuries were inversion injuries (73\%) due to falls $(73 \%)$. At review four fractures were seen that were missed initially. These occurred in significantly older patients requiring symptomatic treatment only. No difference was found in the size of the effusion compared to those without fracture. The average size of effusion was $15 \mathrm{~mm}(7-28 \mathrm{~mm})$ and the STI was 20 (normal 1-15). All but two resumed to their normal activities at six months. Of these two one had a complete anterior talofibular ligament rupture needing surgical stabilisation and the other had no abnormal findings with further investigations and has now resumed to normal activities. Investigations based on the size of effusion would not have selected these two and only two of the missed fractures would have been detected. The functional outcome at six months was not related to the size of effusion. The factors that were related were the size of soft tissue swelling and the STI. In addition we found that those patients who heard a distinctive noise at injury and those treated by plaster took significantly longer to recover. Conclusions-An ankle effusion following injury may indicate a significant injury but it is a poor predictor of the clinical and functional outcome and further investigation based on the size of effusion is not indicated.

Facial $x$ rays in the emergency department

G Little, E Glucksman, R Lenthal

Accident and Emergency Department, King's College Hospital, Denmark Hill, London SES 9RS

Patients with facial trauma usually have radiographs as part of their assessment and initial management in A\&E. However, concern has been expressed that some patients may be having unnecessary and potentially harmful $x$ ray examinations in the A\&E department. A retrospective study was performed to assess the contribution of each radiograph in a standard set of three radiographs [lateral, occipito-mental (OM), occipito-mental $30^{\circ}$ ] to the diagnosis of facial trauma. The $x$ rays of 120 patients with facial trauma were reviewed to determine the proportion of patients with normal and abnormal findings. All 120 patients had undergone varying numbers and types of view, but all had an OM view. The $120 \mathrm{OM}$ views were examined separately by a radiology registrar and an A\&E SHO in order to ascertain the sensitivity and specificity of using the $O M$ view alone in diagnosing facial fractures. With each $\mathrm{OM}$ view the radiology registrar was asked if further views would aid in the radiological diagnosis. The results are discussed and recommendations made about radiographic investigations for patients presenting to $A \& E$ with facial trauma.

Factors associated with heroin overdose G W McNaughton, R L Smith, H C Martindale Accident and Emergency Department, Glasgow Royal Infirmary University NHS Trust, 84 Castle Street, Glasgow G4 OSF

The increase in drug related deaths in Glasgow over the last five years has resulted in considerable media attention. Studies assessing the toxicological aspects of these deaths have reported that heroin has been involved in over $60 \%$ of cases. This study was designed to audit the number and pattern of heroin overdoses requiring acute treatment in a large inner city A\&E department and to specifically identify factors which contributed to the patients attendance. It was a retrospective review of patients treated in resuscitation room following heroin overdose over a one year period (Feb 95-Jan 96).

Results -41 patients were identified ( 31 male, 10 female), mean age 26 years (range 19-39). $A$ review of the individual $A \& E$ record cards revealed four contributory factors: (1) other drugs ingested (35\%); (2) recent release from prison (30\%); (3) abstinence/infrequent drug misuse (25\%); (4) alcohol intoxication (10\%).

Conclusions-Heroin overdoses and heroin related deaths remain a common problem in Glasgow. In this study they accounted for $0.6 \%$ of all resuscitation cases in one year. Action to reduce overdoses in the injecting population should focus on education with regard to the factors identified in this study.
Magnetic resonance imaging of childhood musculoskeletal injuries at the ankle

$\mathrm{R}$ Boyd

Accident and Emergency Department, Booth Hall Children's Hospital, Charlestown Road, Blackley, Manchester M9 $2 A A$

We studied 10 children who presented with a history of an inversion injury of the ankle. The clinical signs elicited on initial examination suggested bony injury. Plain radiographs taken on presentation appeared normal. Magnetic resonance imaging of the injured ankle was successfully performed on all subjects within seven days of initial assessment. The images obtained were interpreted and scored on six pathological criteria. These pathological criteria and their incidence are shown (table).

Pathological criteria and incidence on $M R$ scanning

\begin{tabular}{ll}
\hline Pathology & Incidence \\
\hline Growth plate injury & $50 \%$ \\
Joint effusion & $70 \%$ \\
Periosteal elevation & $70 \%$ \\
Peroneal tendon injury & $40 \%$ \\
Ligamentous injury & $30 \%$ \\
Saphenous venous stasis & $20 \%$ \\
\hline
\end{tabular}

We conclude that children with clinical evidence of bony injury/postinversion injury of the ankle despite normal plain radiographs do indeed have demonstrable bone and soft tissue injury on magnetic resonance imaging.

Duplication and patient delays in the A\&E department

G C Foster, B P McNicholl, B Fisher

Accident and Emergency Departments, Belfast City and Royal Victoria Hospitals, Belfast

Objective-To determine the number of patients attending an $A \& E$ department who could be referred to acute assessment units at triage.

Setting - An urban A\&E department in a teaching hospital.

Design-Prospective audit of all patients attending over a $15 \mathrm{~d}$ period.

Main outcome measures-Source of patient, number of patients suitable to bypass $A \& E$, actual disposal, specialties involved.

Results-Of 1626 patients, 308 (19\%) could have been sent to an acute assessment unit at triage and $85 \%$ of these could have been referred by the triage nurse. Half of the patients were general practitioner referrals. $30 \%$ of the general practitioner referrals were discharged from the A\&E department and $53 \%$ of the self referrals. In all, $57.8 \%$ of all patients were admitted. 
Conclusions-75\% of the patients suitable for triaging to an acute assessment unit are admitted or referred to an outpatient department. The time they spend in an A\&E department delays them getting to their definitive specialty and is a wasteful duplication of resources. Triage should assess whether access to specialist assessment should be through the A\&E department or not. This would reduce A\&E attendances by $19 \%$ and provide a better patient service.

Medical students' attitude towards A\&E as a career

B Duggan, B P McNicholl

Accident and Emergency Departments, Belfast City Hospital and Royal Victoria Hospital, Grosvenor Road, Belfast BT12 6BA

Medical students' attitudes to $A \& E$ were assessed through a questionnaire at their introductory lecture to their final medical year. Thirteen questions were answered involving "yes/no" or tick box answers. 94 of 160 students returned a questionnaire. The majority of students $(83 \%)$ felt $A \& E$ experience would be important for their future career but none chose it as their preferred career. Two thirds of them knew less about a career in $A \& E$ than other major specialties to which they had been attached. They considered A\&E was harder training $(62 \%)$, harder work $(78 \%)$, and more stressful $(93 \%)$ than other specialties. This negative attitude to $A \& E$ as a career may partly explain the difficult in recruiting doctors to the specialty. We believe it is erroneous and that better advice concerning the specialty during attachments to $A \& E$ may improve this outlook.

Scale and cost of preventable trauma deaths

B P McNicholl

Accident and Emergency Department, Royal Victoria Hospital, Grosvenor Road, Belfast, BT12 $6 B A$

Deaths from major trauma were audited in a randomised sample of hospitals in Northern Ireland over a one year period. Trauma deaths were defined by the trauma score and age combination index (TRISS) and by peer review. There were 74 trauma deaths ISS > 15. Preventable deaths ranged from $3-38 \%$ by peer review, depending on the level of panel agreement, or $50 \%$ by TRISS. Median age was 59 years, range 18-91. Following ATLS protocols may have avoided five deaths $(18 \%)$, consultant presence a further three $(11 \%)$. O seven patients whose management errors occurred in the first few hours, four were seen by a trauma team. Large hospitals had the highest preventable death rates and their patients were more often cared for by junior doctors. $98 \%$ of the preventable deaths were predicted to be disabled at the one year had they survived.

Conclusions-ATLS training and protocols for consultant call out may reduce preventable deaths by one third. Further reductions are more difficult to predict. Some of these depend on broader healthcare issues concerning care of the elderly, rehabilitation, and quality of life. Preventing trauma deaths through better trauma care may not be cost-effective. Prevention may be better directed at morbidity and disability.
The effect of different resuscitation fluids on lymphocyte proliferation after haemorrhage

J J Ward, E A M van Rijen, I V Hutchinson, $\star \mathrm{R}$ A Little

North Western Injury Research Centre and *Immunology Research Group, Manchester University, M13 9PT

Haemorrhage can affect many aspects of immune function. Compromised immunity may be one of the factors involved in the development of complications after trauma. One part of the immune system that can be seriously compromised after haemorrhage is the ability of lymphocytes to proliferate in response to stimuli, resulting in poor cellular immune responses. It has also been reported that resuscitation fluids can adversely affect immunity, especially RES function. The aim of this study was to see if lymphocyte proliferation after haemorrhage was affected by the type of resuscitation given. Young adult male Balblc mice (23-27 g) anaesthetised with isoflurane were bled through a cannula placed in the left femoral artery at a rate of 0.1 $\mathrm{ml} / \mathrm{min}$ until $40 \%$ of their blood volume was removed; $30 \mathrm{~min}$ after the end of haemorrhage. they were resuscitated with their shed blood or an equal olume of hydroxyethyl starch or Haemaccel, or $2 \times$ volume of Ringer's lactate. Sham operated animals received surgery but no haemorrhage/ resuscitation. At 2,24 , or $48 \mathrm{~h}$ after the end of haemorrhage, animals were killed and their spleen and mesenteric lymph nodes removed. A single cell suspension was made of each. $2 \times$ $10^{5}$ cells were incubated with or without the non-specific mitogen concanavalin $\mathrm{A}$ for $48 \mathrm{~h}$ at $37^{\circ} \mathrm{C}, 5 \% \mathrm{CO}_{2} .1 \mu \mathrm{Ci}$ of ${ }^{3} \mathrm{H}$-thymidine was added and the cells cultured for another $24 \mathrm{~h}$. The cells were harvested and the uptake of ${ }^{3} \mathrm{H}$-thymidine was measured on a scintillation counter. Data were analysed by anova. In all groups there was a depression of splenocyte proliferation at $24 \mathrm{~h}$, which normalised by 48 h. There were no significant changes in mesenteric lymph node cell proliferation in any group over this time course. Different resuscitation fluids seem to exert no effect on lymphocyte proliferation beyond that already caused by the surgery and anaesthesia in this model. Different lymphocyte populations behave differently after haemorrhage and resuscitation. Thus while it is clear that certain aspects of immune function can be altered by the trauma of surgery, there is no evidence in the present study that resuscitation fluids further modify such responses.

Tissue injury attenuates the hypotension of haemorrhagic shock but worsens the metabolic insult

B A Foëx, R A Little, E Kirkman, T Rainey

North Western Injury Research Centre, Stopford Building, Oxford Road, Manchester M13 9PT

Tissue injury attenuates the biphasic heart rate response and the hypotension induced by blood loss. These apparent physiological.benefits are not matched by improvements in survival. This apparent paradox merits further investigation. Increasing plasma lactate concentrations are considered a hallmark of shock. We measured plasma lactate levels in a porcine model of haemorrhagic shock and of haemorrhagic shock on a background of nociceptive afferent nerve stimulation (which mimics the effects of tissue injury). 10 animals were studied in each of four groups: $\mathrm{C}$, surgical controls; $\mathrm{H}, 30 \%$ total blood volume haemorrhage; HNS, $30 \%$ haemorrhage on a background of nociceptive afferent nerve stimulation; and NS, nerve stimulation alone. An alphaxolone/alphadolone infusion was used for anaesthesia. The animals were bled over $30 \mathrm{~min}$ and the shed blood was reinfused after $30 \mathrm{~min}$ of shock. Nerve stimulation was started before haemorrhage in HNS and continued throughout the 90 min study.

Results-HNS produced a greater tachycardia and an attenuation of the hypotension seen with $\mathrm{H}$. CI and $\mathrm{DO}_{2} \mathrm{I}$ both fell to around $50 \%$ of prehaemorrhage values in $\mathrm{H}$ and $\mathrm{HNS}$ but $\mathrm{VO}_{2} \mathrm{I}$ was unchanged, as OER increased from $29 \%$ to $65 \%$ in $\mathrm{H}$ and from $27 \%$ to $64 \%$ in HNS. Resuscitation reversed all the changes except the tachycardia. Plasma lactate after resuscitation reached $4.8 \mathrm{mmol}$ in HNS compared to $2.3 \mathrm{mmol}$ in $\mathrm{H}$. Taking the rise in lactate over the course of the study $(\Delta \mathrm{lac})$ as an index of the severity of shock, $\Delta$ lac was greater for HNS than for $\mathrm{H}(\mathrm{P}=0.0008)$.

Conclusions-There is evidence that HNS results in more severe shock than $\mathrm{H}$ despite the fact that global cardiovascular indices seem to be no worse. It is likely that disturbances in regional blood flow result in a greater metabolic insult in HNS than in $\mathrm{H}$.

Oscillating saw injuries during removal of plaster

M Z Ansari, S Swarup, R Ghani

Alexandra Healthcare NHS Trust, Woodrow Drive, Redditch B98 $7 U B$

Objective-To assess the incidence of injuries to patients who have had plaster cast removed by oscillating circular saw at The Alexandra Hospital, Redditch, and to recommend measures to avoid such injuries.

Setting-A\&E department and plaster room. Methods-The record of each patient who had their plaster removed was kept in plaster roam and later studied.

Results - 3875 plaster casts were removed in one year (1995); 28 patients $(0.72 \%)$ sustained abrasions or burns over the skin.

Conclusions-Removal of plaster cast by an inexperienced, ill trained user or blunt saw blade was the identified cause of injury. Strict protocols were required and introduced at The Alexandra Hospital to avoid litigation.

An evaluation of paramedic activities in prehospital trauma care

T H Rainer, K P G Houlihan, C E Robertson, D Beard, J M Henry, M W G Gordon Accident and Emergency Department, Royal Infirmary of Edinburgh, Lauriston Place, Edinburgh EH3 9YW

Objectives-To identify the relative effects of ambulance paramedics and technicians on prehospital trauma care.

Design-A prospective review was performed of ambulance and hospital records from $1 / 8 / 1993$ to $31 / 7 / 1995$. Information sources included prehospital ambulance data, A\&E notes, and inhospital records. Data collection included prehospital times, patient triage category, and initial destination after leaving the A\&E department.

Setting-The Royal Infirmary of Edinburgh and its primary response catchment area 
served by the South-East Region of the Scottish Ambulance Service central control room. Subjects-1090 patients who met the entry criteria for the Scottish Trauma Audit Group and Major Trauma Outcome Study (UK). Main outcome measures-Mortality, time in intensive care and in hospital.

Results - The groups of patients were similar with regard to age, sex and injury severity as measured by ISS scores. Paramedics treated significantly more penetrating trauma, patients whose injuries were defined as major (that is, road traffic accidents, assaults, and falls greater than $2 \mathrm{~m}$ ), and patients with abnormal RTS scores. Paramedics spent significantly longer at scene than ambulance technicians ( $19 v 16 \mathrm{~min}, \mathrm{P}<0.001$ ) but there was no difference in total prehospital times between the groups. Paramedics direct a significantly greater proportion of patients to the resuscitation room from each ISS and RTS group. Significantly more of these patients go to theatre, intensive care, or the mortuary $(10 \% v 4 \%, P<0.001)$. Length of hospital stay and in intensive care was identical in both groups. In all, 36 patients died The mortality was no different for patients treated by paramedics than by technicians ( $4 \%$ v $3 \%, \mathrm{P}=\mathrm{NS}$ ).

Conclusions-Paramedics may deliver an improved process of care but these activities do not translate into objective improvements in mortality or length of stay in hospital or intensive care.

A survey of plastic bullet injuries presenting to hospital accident and emergency departments during a week of civil disturbance in Northern Ireland

S J McBride, J Steele, J Kelly

Accident and Emergency Department, Mater Hospital Trust, Crumlin Road, Belfast BT14 $6 A B$

Plastic bullets have been available to police forces as a method of riot control throughout the UK since 1988 but have been exclusively used in Northern Ireland by the security forces since their introduction in 1973 , completely replacing the less accurate rubber bullet in 1975. Their use has been controversial and has been associated with fatalities. The plastic bullet is a projectile made of polyvinyl chloride (PVC). It is a cylinder measuring $10 \times 3.7 \mathrm{~cm}$ and weighs $135 \mathrm{~g}$. It is discharged from the riot control gun at low muzzle velocity to travel on its long axis to hit the target end on. During the week 8 to 15 July 1996 widespread serious public disorder occurred in Northern Ireland and some 6000 plastic bullets were fired. We report a series of 177 patients who attended A\&E departments in eight hospitals (seven in Northern Ireland and one in the Irish Republic) during this period. For 176 of these patients 183 separate injuries attributed to plastic bullets have been recorded. There were 39 injuries to head and neck, 31 to upper limb, 26 to chest, 13 to abdomen, and 74 to lower limb. ISS scores were calculated for these 176 patients. These scores ranged from 1 to 18.45 patients required hospital admission of whom four required intensive care treatment. There were no fatalities.
Morphine reverses the bradycardia and hypotension associated with severe haemorrhage

M Ohnishi, E Kirkman ${ }^{\star}$, H W Marshall, R A Little

North Western Injury Research Centre, Stopford Building, University of Manchester, M13 9PT and ${ }^{\star}$ Department of Biological Sciences, Science Laboratories, University of Durham, DH1 3LE

The response to progressive simple haemorrhage (blood loss in the absence of major tissue damage, eg gastrointestinal haemorrhage) is biphasic in man and rat: an initial tachycardia while blood pressure is maintained by the baroreflex followed by a vagally mediated bradycardia and hypotension due to the activation of a second depressor reflex. The latter phase can be attenuated by prior administration of morphine. The aim of this study was to determine whether the bradycardia and hypotension could be reversed by morphine, once they had been established. Experiments were performed on two groups of male Wistar rats (236-258 g). Stainless steel guide cannulas were implanted into the lateral cerebral ventricle (icv) under pentobarbitone anaesthesia $(60 \mathrm{mg} / \mathrm{kg}$ ip) $7-14 \mathrm{~d}$ before the study. On the day of the study anaesthesia was induced with isoflurane $\left(3.5 \%\right.$ in $\left.\mathrm{O}_{2}\right)$ and maintained with alphadolone/alphaxalone (16-18 $\mathrm{mg} / \mathrm{kg} / \mathrm{h}$ iv) The electrocardiogram was recorded from needle electrodes attached to the skin of the ventrum, blood pressure from the ventral tail artery, and body temperature with a recta probe. Body temperature was maintained constant at $38.0(\text { SEM } 0.0)^{\circ} \mathrm{C}$ using external heating. Both groups received a haemorrhage of $40 \%$ total blood volume (BV; estimated as $6.06 \mathrm{ml} / 100 \mathrm{~g}$ body weight) at $2 \% \mathrm{BV} / \mathrm{min}$ After the loss of $26 \% \mathrm{BV}$, bradycardia and hypotension were established equally in both groups (table). Group I $(n=8)$ then received $0.9 \%$ saline $(20 \mu \mathrm{l} \mathrm{icv})$ while group II $(n=10)$ received morphine $(10 \mu \mathrm{g}$ in $20 \mu \mathrm{l}$ icv). In group $I$, heart rate (HR) and mean arteria blood pressure (MBP) continued to fall, while the bradycardia was completely reversed and the hypotension attenuated in group II following treatment with morphine (table at foot of page) Conclusions - These results indicate that morphine can completely reverse the bradycardia and partially reverse the hypotension associated with severe haemorrhage. However, any benefit may be more apparent than real since morphine reverses a cardioprotective reflex and produces a pattern of response reminiscent of that induced by injury, which is known to be deleterious.

The effects of calcium containing intravenous resuscitation fluids on platelet aggregation in whole blood in vitro

P A Evans ${ }^{1}$, M S Riyati ${ }^{1}$, J Glenn ${ }^{3}$, W Madira ${ }^{2}$, $S$ Heptinstall ${ }^{3}$

'Accident and Emergency Department, Leicester Royal Infirmary, Leicester LE1 5WW

\begin{tabular}{lllllll}
\hline & Group I (saline) & \multicolumn{3}{l}{ Group II (morphine) } \\
\hline $\begin{array}{lllllll}\text { Haemorrhage } \\
\quad \% \text { BV) }\end{array}$ & $0.0(0.0)$ & $26.7(0.2)$ & $40.0(0.0)$ & $0.0(0.0)$ & $26.4(0.2)$ & $40.0(0.0)$ \\
HR (beats/min) & $420(7)$ & $353(22) \ddagger$ & $291(6) \ddagger$ & $408(10)$ & $340(13) \ddagger$ & $399(16)^{\star}$ \\
MBP (mm Hg) & $98(3)$ & $43(6) \ddagger$ & $22(1) \ddagger$ & $101(3)$ & $39(2) \ddagger$ & $51(8) \ddagger^{\star}$ \\
\hline
\end{tabular}

$\star \mathrm{P}<0.05 v$ corresponding group I value (repeated measures analysis of variance, ANOVA). $\ddagger \mathrm{P}<0.05 v$ prehaemorrhage values ( $0 \% \mathrm{BV}$ ) (repeated measures ANOvA)
${ }^{2}$ Department of Biochemistry, Leicester Royal Infirmary, Leicester LE1 5WW;

${ }^{3}$ Cardiovascular Medicine, University Hospital,

In the traumatised patient serum ionised calcium levels tend to fall and those receiving calcium containing fluids (eg, Haemaccel = $6.2 \mathrm{mM} \mathrm{Ca}{ }^{2+}$ ) have increased bleeding times. We investigated to what extent the serum ionised calcium levels rose in these traumatised patients receiving Haemaccel, and the effect of certain colloids on platelet aggregation in whole blood in vitro.

Methods-Serial serum ionised calcium levels were measured over $24 \mathrm{~h}$ in 19 severely traumatised patients who had received Haemaccel. We also compared the effects of platelet aggregation in hirudinised normal whole blood in vitro of Haemaccel, Gelofusine, Gelofusine to which $6.2 \mathrm{mM} \mathrm{CaCl}_{2}$ was added, and $\mathrm{CaCl}_{2}$ alone. The agonists used were $0.3 \mu \mathrm{M}$ ADP, $0.3 \mu \mathrm{M}$ PAF, $1 \mu \mathrm{g} / \mathrm{ml}$ collagen, $0.3 \mu \mathrm{M} U 46619,1 \mathrm{mg} / \mathrm{ml}$ ristocetin, and $100 \mu \mathrm{M}$ adrenaline. Platelet aggregation was measured using the Ultra-flow-100 counter.

Results-At all time points the mean ionised calcium levels rose and were significantly higher than basal values; in some patients there was significant hypercalcaemia. Both Haemaccel and $\mathrm{CaCl}_{2}$ inhibited platelet aggregation in response to all agonists. However, the inhibition seen with Haemaccel was greater than that seen with calcium alone in all instances, except when collagen was used as the aggregating agent. This suggests that Haemaccel contains other factors apart from calcium which add to its inhibitory effects. Gelofusine had little effect on the aggregation induced by all agents. Addition of calcium to Gelofusine resulted in inhibition of aggregation induced by most agents, but the degree of inhibition was not greater than that seen with calcium alone. In the case of ristocetin, Gelofusine, like Haemaccel, abolished the agglutination that occurred.

Conclusions-Haemaccel causes marked inhibition of platelet aggregation in vitro in response to all agonists investigated. The inhibitory effect of colloids on platelet aggregation may adversely affect normal haemostatic mechanism in trauma patients. These effects are enhanced when calcium is added to both colloids and crystalloids and this may be clinically significant.

Review of PSVT management in the accident and emergency department: the use of adenosine and vagal treatment P A Evans

Accident and Emergency Department, Leicester Royal Infirmary NHS Trust, Leicester LE1 $5 W W$

No previous study of $A \& E$ management of PSVT in the UK has been published.

Design-Prospective A\&E and medical records of patients from December 1994 to Nottingham NG7 2UH 
March 1996 presenting to the A\&E department with PSVT, treated with vagal methods or adenosine were entered into the study. A consultant cardiologist retrospectively assessed the ECG and rhythm strips.

Results-There were 108 presentations: 57 received vagal manoeuvres, 89 adenosine. The SHO identified the initial rhythm as NCT (17), BCT (2), SVT (60), AF (8), ST (1), VT (2), unsure (6), none noted (12). A cardiologist assessed the rhythms: junctional nodal reentrant tachycardia and junctional reentrant tachycardia (JT) (44), AF (19), VT (2), atrial flutter (5), sinus tachycardia (1), unsure 1 (total 65); 11 no ECG; results awaited, 26.

Successful treatment of junctional tachycardiatwo spontaneous, three vagal of 28 attempted; 30 adenosine of 37 attempted; 23 were admitted and three had recurrence requiring drugs. Adenosine side effectsoccurred in 13 patients: chest pain (5), dyspnoea (2), hypotension (2), flushing (3), nausea (1), headache (1), bronchospasm (1); three of these effects occurred in one patient.

Treatment of atrial fibrillation (19)-One spontaneous, none resolved with vagal actions or adenosine. One patient, a 24 year old male with Wolf-Parkinson-White syndrome had ventricular fibrillation precipitated by carotid massage treated successfully with defibrillation.

Conclusions-(1) Adenosine is used too often with atrial fibrillation; fine irregularity is not recognised. (2) Periarrest guidelines need repeat teaching and specific department guidelines are advised. (3) Carotid sinus massage is not without risk it is suggested that it should only be performed on a monitored patient with full ALS facilities present.

Using psychomotor skill teaching technique to teach the measurement of stroke distance for the detection of haemorrhage in the emergency department

J Greenbaum', J M Hanson', E Kirkman', P Driscoll ${ }^{3}$, M A Horan ${ }^{4}$, $S$ Hollis ${ }^{3}$

Accident and Emergency Department, Royal Preston Hospital, Fulwood, Preston PR2 9HT ${ }^{2}$ North Western Injury Research Centre and Departments of Emergency and ${ }^{4}$ Geriatric Medicine, University of Manchester

Blood pressure and heart rate are poor early indicators of haemorrhage. However, stroke volume falls early in haemorrhage and can be assessed ultrasonically as stroke distance (SD). SD is a better early indicator of blood loss than blood pressure or heart rate during simulated haemorrhage (lower body negative pressure, LBNP) and blood donation. To be useful in the emergency department an operator must be taught to detect changes in SD following minimal training. The aim of this study was to show the effectiveness of the psychomotor skill training technique (used in advanced life support courses) in teaching a practical skill. 23 students were given background information on the ultrasound technique one week before the practical session. At the beginning of the practical session they were assessed using an MCQ to ensure they had assimilated the information. They were then taught for $1 \mathrm{~h}$ using the psychomotor skill training technique to measure SD using a portable Doppler monitor (Deltex Medical). The skill was assessed by monitoring volunteers subjected to LBNP: SD was measured at a LBNP of 0,20 , and $40 \mathrm{~mm} \mathrm{Hg}$ by each student followed by the instructor, blinded to each other's measurements. The instructor showed a fall in SD with each step of LBNP $(15.1 \%, 30.1 \%$, respectively $0-20 \mathrm{~mm} \mathrm{Hg}$, 0-40 mm Hg LBNP). 22 students showed a fall in SD between 0 and $20 \mathrm{~mm} \mathrm{Hg}$ (which is equivalent to a blood loss of approximately $300 \mathrm{ml}$ ), while they all showed a fall in SD between 0 and $40 \mathrm{~mm} \mathrm{Hg} \mathrm{LBNP.} \mathrm{Thus} \mathrm{the}$ measurement of SD using the portable Doppler ultrasound technique can be taught in $1 \mathrm{~h}$. This shows that the psychomotor skill training technique is an educational tool which should be considered when teaching practical skills in medicine.

Alteration in leucocyte adhesion molecule expression following moderate and major trauma

R A Cocks, Y F Chan

Accident and Emergency Medicine Academic Unit, The Chinese University of Hong Kong, Room G05/06, Cancer Centre, Prince of Wales Hospital, Shatin, NT, Hong Kong

An understanding of the mechanisms of postinjury leucocyte trafficking is essential to the development of future treatments aimed at preventing infection and multiple organ failure. While the changes in circulating leucocyte numbers after trauma are clear, very little is known about the cellular and molecular events which result in mobilisation of these cells. We have studied the postinjury expression of lymphocyte, monocyte, and neutrophil CD11a (LFA1), CD11b, Cd11c, CD29 ( $\beta-1$ integrin) and CD62L (L-selectin) in 29 patients, 10 of whom had suffered major trauma (ISS 16 or greater). We also studied 42 normal control subjects for comparison.

Methods-Blood samples were taken from 29 patients within $2.5 \mathrm{~h}$ of injury into EDTA anticoagulant. Fluorescent labelled monoclonal antibodies to each adhesion molecule were used to detect both the percentage of each cell line expressing each molecule and the strength of expression (mean channel fluorescence) on a Coulter Epics XL flow cytometer.

Results-Monocytes, neutrophils, and lymphocytes in the trauma patients showed significantly increased mean channel fluorescence expression of L-selectin $(P<0.0005$, 0.005 , and 0.05 , respectively). Neutrophils showed a significantly decreased level of expression of CD11a and CD11c, but the lower level of CD11b expression with respect to the controls did not reach significance. Monocytes showed a significant decrease in CD11a expression, but the reduction in ymphocyte CD11a expression was nonsignificant.

Conclusions - There is a reduction in CD11a expression after trauma which may play an important role in the demargination of neutrophils and monocytes. The strong increase in L-selectin expression in all cell lines was unexpected, and is potentially important in that this molecule supports rolling behavour in neutrophils, and is the molecule which is in the most advantageous position to contact the endothelium at the site of injury.

First and second median (range) plasma potassium and glucose (mmol/l) values

\begin{tabular}{llll}
\hline Plasma values & 1st sample & 2nd sample & Pvalue $^{\star}$ \\
\hline Potassium & $3.6(2.2-5.1)$ & $3.8(3.1-5.3)$ & 0.0184 \\
Glucose & $6.1(2.7-13.1)$ & $6.4(4.6-8.5)$ & 0.0392 \\
\hline
\end{tabular}

$\star$ Wilcoxon signed rank test.

Serum potassium and glucose following non-thermal trauma in children

T H Rainer', C Barclay ${ }^{1}$, T Beattie', P Crofton ${ }^{3}, \mathrm{~N} \mathrm{McIntosh}^{2}, \mathrm{M} \mathrm{McNicol}^{3}$, K Sedowofia ${ }^{2}$, R Stephen ${ }^{2}$

Department of Accident and Emergency Medicine, Royal Hospital for Sick Children, Edinburgh; 'Department of Child Life and Health, University of Edinburgh, Edinburgh; ${ }^{3}$ Department of Paediatric Biochemistry, Royal Hospital for Sick Children, Edinburgh

Little is known about the hormonal and electrolyte changes following moderate trauma in children. Chance observation indicates that serum potassium falls and serum glucose rises in some children but not in others. The effect of analgesia on hormonal and electrolyte factors following trauma in children is also poorly understood. Assumptions are often made that paediatric responses mimic adult responses and that electrolyte imbalances such as hypokalaemia require potassium supplementation.

Objective-To investigate the relation between serum potassium and glucose following moderate trauma in children.

Methods-Ethics approval was obtained from Lothian ethics committee for two blood samples to be taken from children presenting to the A\&E department of The Royal Hospital for Sick Children following trauma. Intravenous (IV) access was gained according to normal protocol and one aliquot of blood was taken on admission and before the administration of IV analgesia. A second sample was taken several hours later before the induction of anaesthesia. No patient received IV fluid between the two samples. Diabetics were excluded. Fisher's exact, $\chi^{2}$, Wilcoxon signed rank tests were used where indicated.

Results-There were 34 children (27 males), aged 11 months to 14 years (median 8 years) with a paediatric trauma score (PTS) ranging from 6 to 12 (median 9), an injury severity score (ISS) ranging from 1 to 11 (median 9), and a maximum abbreviated injury score (MAIS) of 1-3. The table (at foot of page) shows differences in potassium and glucose between the first and second sample. In seven cases the initial potassium level was outside the normal range $(<3.5 \mathrm{mmol} / \mathrm{l} ; \mathrm{P}=0.02)$, the lowest of which was $2.2 \mathrm{mmol} / \mathrm{l}$. All had corrected to "normal" by the second sample except for two, which were 3.1 and 3.4 $\mathrm{mmol} / \mathrm{l}$. There was no correlation between ISS, MAIZ, PTS, and potassium and glucose on the first or second sample. However, potassium correlated with glucose $(r=0.535, \mathrm{P}=$ 0.0076 ) on the later sample.

Conclusions-Stress following trauma alters the normal hormonal and electrolyte patterns of children. Initial hypokalaemia probably does not require potassium supplementation as the changes are temporary and quickly correct either with time or appropriate analgesia. 
Stress responses following mild to moderate trauma in children

T H Rainer' ${ }^{1}$, C Barclay ${ }^{2}$, T Beatti ${ }^{1}$, P Crofton ${ }^{3}$, N McIntosh', M McNicol ${ }^{3}$, K Sedowafia ${ }^{2}$, R Stephen ${ }^{1}$

'Department of Accident and Emergency, Royal Hospital for Sick Children, Edinburgh; ${ }^{2}$ Department of Child Life and Health, University of Edinburgh, Edinburgh; ${ }^{3}$ Department of Paediatric Biochemistry, Royal Hospital for Sick Children, Edinburgh

Stress responses following trauma in children have been poorly characterised and little is known regarding the hormonal and electrolyte changes following moderate trauma in this group. The effect of analgesia on hormonal and electrolyte factors following trauma in children is also goody understood. Assumptions are often made that paediatric responses mimic adult responses. Consequently adult protocols are used in the treatment of paediatric patients.

Objective-To characterise the hormonal and electrolyte changes following the stress of moderate trauma in children.

Methods-Ethics approval was obtained from the local ethics committee to take two aliquots of blood from children admitted following trauma - the first on admission and just before the administration of amnesia, and the second several hours later before general anaesthesia. Over three months 34 children (27 males) aged 11 months to 14 years (median 8 years) with non-thermal limb injuries attended the A\&E department at The Royal Hospital for Sick Children. The paediatric trauma score (PTS) ranged from 6 to 12 (median 9), the injury severity score (ISS) ranged from 1 to 11 (median 9), and the maximum abbreviated injury score (MAIS) ranged from 1 to 3 . No patient received intravenous (IV) fluid before the second blood sample. Correlation was sought between PTS, ISS, MAIS, and plasma levels of adrenaline (ADR), noradrenaline (NOR), cortisol, angiotensin II (ANG II) and arginine vasopressin (AVP). Fisher's exact and Wilcoxon signed rank tests were used where indicated.

Results-Correlations on the first sample, taken 5-186 minutes after injury, were found on the following: ADR with MAIS $(r=341, \mathrm{P}$ $=0.0517)$. ADR with PTS $(r=0.394, \mathrm{P}=$ 0.0225 ). Further correlations on the second sample, taken 60-260 min after admission, were: cortisol and ISS $(r=0.448, \mathrm{P}=0.0108)$; cortisol and MAIS $(r=0.392, \mathrm{P}=0.0283$ ) The table (at foot of page) shows the hormonal changes with time and intervention. Conclusions-Stress following trauma alters the normal hormonal patterns of children. Cortisol and adrenaline correlate with injury severity and show the greatest change with time and/or intervention.

First and second median (range) hormonal plasma values

\begin{tabular}{llll}
\hline & 1st sample & 2nd sample & Pvalue $^{*}$ \\
\hline Cortisol (nmol/l) & $634(1.27-916)$ & $410(30-863)$ & 0.0008 \\
Noradrenaline (nmol/l) & $2.27(0.42-5.06)$ & $2.16(0.82-4.24)$ & 0.0937 \\
Adrenaline (nmol/l) & $1.05(0.09-66.3)$ & $0.65(0.02-61.8)$ & 0.0011 \\
Angiotensin II (pmol/l) & $29.45(9.9-56.5)$ & $25.4(6.5-86.1)$ & 0.2597 \\
Arginine vasopressin (pmol/l) & $6.5(0-29.2)$ & $2.95(1-27.1)$ & 0.1428 \\
\hline
\end{tabular}

*Wilcoxon signed rank test.
Training and willingness of bystanders to

T H Rainer, K Dalziel

Directorate of Accident and Emergency Medicine, Glasgow Royal Infirmary, Glasgow

Ischaemic heart disease remains a major cause of sudden cardiac death in the western world in general and in the west of Scotland in particular. Two principal factors have been shown to improve outcome directly following cardiac arrest if done early-defibrillation and basic life support (BLS). Factors that improve the delivery of BLS and defibrillation will indirectly improve outcome. Although there has been significant improvement in survival from prehospital cardiac arrest in the east of Glasgow since the early 1980s, the rate of bystander CPR remains poor. We evaluated the perceived ability and willingness of people attending A\&E to perform BLS.

Methods and results-This prospective observational survey, based in the $A \& E$ departmen at Glasgow Royal Infirmary, resulted in 718 completed questionnaires in a one week period. Training in BLS was not related to age although significantly more men had received training than women $(179 / 310,58 \%: 165 / 393$, $42 \% ; \mathrm{P}<0.001) .72$ non-clinical NHS employees $(57 \%)$ were not trained in BLS nor were 306 non-NHS subjects (65\%). 107 respondents $(55 \%)$ who had close relatives with the past history of myocardial infarction had received no training in BLS. Eight social workers and home helps (50\%), 20 students and teachers (54\%), 20 catering, domestic and porters $(77 \%)$, and 11 public transport drivers $(92 \%)$ had received no training in BLS. Training appeared to make no difference to the willingness of respondents to perform CPR on relatives, friends, and strangers However, those who had received training were more certain about whether or not they would perform the procedure. 23 clinical NHS personnel (48\%), 70 non-clinical NHS personnel (57\%), 246 non-NHS subjects $(55 \%)$, and 10 ambulance personnel $(29 \%)$ indicated that they would perform BLS rather than call for an ambulance as the first action in an non-EMS out of hospital cardiac arrest. Conclusions - Large numbers of out of hospita and hospital based personnel remain untrained in BLS. Many ambulance and clinica NHS personnel do not appear to appreciate the value of calling an ambulance early so that early defibrillation may be implemented.

An examination of the accident and emergency management of deliberate self harm: implications for service planning and provision

M S Dennis, Beach, P A Evans, A Winston, $T$ Friedman

Division of Psychiatry for the Elderly, University of Leicester, Leicester General Hospital, Gwendolen Road, Leicester LE5 4PW peform basic life support
Deliberate self harm (DSH) is a frequent cause of acute medical admission, but many patients are discharged directly from $A \& E$. To assist general hospitals in improving services the Royal College of Psychiatrists recently produced a consensus statement on standards of service for the general hospital management of adult deliberate self harm. Using an instrument based upon this statement, we retrospectively examined the management of $\mathrm{DSH}$ at the Leicester Royal Infirmary A\&E department over a 12 month period. There were 934 episodes of DSH involving 854 patients. The mean age was 32 (SD 14.2) years, with an even sex distribution (48\% male, $52 \%$ female). Overdose was by far the most common method of DSH $(91.5 \%$ of cases). Information concerning suicide intent was documented in $70 \%$ of cases, and psychiatric history in $67 \%$. Less information was recorded for medical history $(50 \%)$, mental state $(51 \%)$, recent stress $(55 \%)$, previous DSH (47\%), and only $23 \%$ had an assessment of risk of further DSH. Very little was recorded concerning alcohol or substance misuse. In 291 cases ( $31 \%$ ), the patient was discharged directly home by $A \& E$ medical staff, and 50 of these were referred for phychiatric outpatient follow up. $210(23 \%)$ were referred for specialist assessment in the department, and $46 \%$ admitted to medical/ surgical wards. The frequency with which information was recorded varied significantly between outcome groups. At night $A \& E$ staff were far more likely to discharge a patient home themselves than refer for specialist assessment $(P=0.0003)$.

Conclusions-With over half the sample not admitted, the responsibility for an adequate risk assessment lies with A\&E medical staff. The study reveals a need for improved planning and delivery of services. Various measures are now being implimented: training in the assessment of DSH, the use of preprinted checklists for risk assessment, and the extension of the working hours of the local deliberate self harm team. These findings are also discussed from a national perspective.

The use of the Ottawa ankle rules by nurse practitioners

C J Mann

Department of Accident and Emergency, Derriford Hospital, Plymouth PL6 8DH

Aim-The Ottawa ankle rules used in other centres by doctors have been shown to reduce the number of ankle $x$ rays without missing any fractures. This study assesses the use of the Ottawa ankle rules by nurse practitioners to determine whether they could be applied as effectively as when used by doctors.

Methods - 665 patients over the age of 16 who presented within $24 \mathrm{~h}$ of an ankle injury wer assessed by a nurse trained in the use of the Ottawa ankle rules. Where indicated the nurs sent the patient for an $x$ ray. 700 patients in the same period were seen by doctors who had not received training in these rules. Al patients were subsequiently examined by doctor and an $x$ ray ordered if the doctor considered it appropriate. After three months the doctors were similarly trained in the use of the rules. Data on the reduction in numbers of $x$ rays following this are being collected and will be available for presentation. 
Results -665 patients were assessed by a nurse practitioner. $72.6 \%$ were $x$ rayed. Of the 700 patients seen by a doctor alone, $91 \%$ were sent for $x$ ray $(P=0.001)$. No fractures were missed by the nurse practitioners.

Conclusions-Nurse practitioners trained to use the Ottawa ankle rules can accurately determine which patients require a radiograph of the ankle. A comparison between the percentage of patients $x$ rayed by nurses and doctors after the latter had been taught to use the Ottawa rules will be presented.

\section{Prehospital diazepam: an adult of use} H R Guly

Accident and Emergency Department, Derriford Hospital, Plymouth, Devon

The conventional management of prolonged seizures involves parenteral administration of a short acting benzodiazepine. Paramedic crews working for the Westcountry Ambulance Service Trust have recently started giving rectal diazepam to people who are fitting, according to an agreed protocol.

Methods-We undertook a retrospective audit of all patients brought to our hospital by ambulance who were diagnosed as having suffered a fit. We examined the ambulance and casualty cards of all such patients attending over a time period spanning the six months before and the six months after the introduction of diazepam on the ambulances. We recorded the following information for each patient: date of attendance, age, sex, and whether known to have epilepsy; whether a fit was witnessed by a paramedic; whether anticonvulsant was given in the community and if so by whom (carer or ambulance crew); number of fits and duration of these; length of time the ambulance was at the scene; baseline observations on arrival in hospital and whether the patient was still fitting; disposal of the patient and the final diagnosis of the cause of the fits.

Results-Over the 12 month period we identified 328 patients brought to hospital by ambulance in whom a diagnosis of fits was made. Of these, 51 had been given anticonvulsant before arriving in hospital. The patients who were given anticonvulsant by the ambulance personnel took significantly longer to reach hospital, and were more likely to be admitted overnight than those given no drug. Those treated by the ambulance crews had a significantly lower blood pressure, Glasgow coma score, and respiratory rate on arrival at hospital than those not treated. There was no difference in the pulse rate between the two groups. One patient suffered a respiratory followed by cardiac arrest after being given diazepam by an ambulance paramedic.

Conclusions-We do not think there is enough evidence to support the use of diazepam by ambulance crews in the catchment area of our hospital.

A prospective study of the effect on door to needle time and outcome of different ways of giving thrombolysis treatment to patients with $M I$

M Sakr, J Koch, J Daly, J Eclhause, F Morris
Accident and Emergency Department, Northern General Hospital, Sheffield S5 $7 A U$

In the 1960 s and 1970 s trials evaluated streptokinase treatment in myocardial infarction (MI); however, it was given on the premise that afterload and viscosity would be reduced. In 1976 Chazov gave intracoronary streptokinase to a patient in Russia. In 1973 Rentrop in Germany gave intracoronary streptokinase to a patient who had developed an acute coronary thrombosis during routine cardiac catheterisation. The thrombus was lysed and reperfusion achieved. In 1986 the first GISSI trial in which 11706 patients were randomised to either streptokinase or critical therapy showed a highly significant reduction in mortality. Pooled evidence from recent research showed that the earlier the thrombolysis is given the better the outcome is regarding mortality and left ventricular function.

Aims-(1) To examine onset to needle time and door to needle time of thrombolysis in Sheffield hospitals; (2) to assess route of admission in relation to door to needle time, accuracy of diagnosis, and safety.

Methods-All patients admitted to the two major teaching hospitals of Sheffield are followed up if they presented with ischaemic sounding chest pain. Patients who are proved to have developed or presented with MI are followed in detail and their door to needle time, complications related to thrombolysis, survival, and reasons for delay are documented. Patients presenting with MI who did not receive thrombolysis are followed and the reasons why they did not receive thrombolysis documented.

Subjects-Every patient admitted with ichaemic chest pain was considered eligible for inclusion but only those with MI within $12 \mathrm{~h}$ were actually included. Patients excluded: patients who developed MI after $12 \mathrm{~h}$; silent MI; interhospital referral; collapse.

Procedure-Patients are followed up in CCU and wards, initial diagnosis documented, and diagnosis on discharge documented. Inhospital mortality and mortality after one month is documented.

Prospective trial of Entonox and intraarticular lignocaine for acute anterior shoulder dislocation

P Gleeson, C A Graham, A Meyer

Accident and Emergency Department, Royal Infirmary of Edinburgh, Lauriston Place, Edinburgh EH10 6QD

Objective-To assess the relative analgesic effects of Entonox and intra-articular lignocaine in patients presenting with acute anterior shoulder dislocation.

Patients and methods-A prospective randomised trial was conducted between March and July 1996. All patients over 16 presenting with anterior glenohumeral dislocations without an associated fracture were considered for inclusion in the study. Patients received either
Entonox through a Mapleson C circuit or an intra-articular injection of $10 \mathrm{ml} 1 \%$ plain lignocaine. Objective verbal pain scores were obtained before and after analgesia and also after reduction. The Milch and external rotation manoeuvres were the reduction methods of choice and the pain experienced during reduction was assessed. Results were analysed using the Wilcoxon rank sum and paired Student $t$ tests.

Results-There were 31 patients in the study, 15 of whom received intra-articular lignocaine and the other 16 received Entonox. The mean ages and sex distribution were comparable for both groups. The mean pain scores are shown in the table (at foot of page). 11 patients in each group described the reduction manoeuvre as being either slightly painful or not painful at all. Four patients in the intra-articular group required supplemental analgesia to facilitate reduction while only one patient in the Entonox group required intravenous sedation/ analgesia.

Conclusions-Entonox given through a Mapleson $\mathrm{C}$ circuit significantly reduces pain scores in patients with acute anterior shoulder dislocations, allowing comfortable reduction in the majority of cases. In comparison, intraarticular lignocaine is less effective.

Effect of a standard one unit blood donation on stroke distance, blood pressure, and pulse rate in a cohort of elderly blood donors

L Bethune, J Stuart, M Saab, J Hanson, A Clayson, P Randall

$A \mathcal{E} E$ Department, Manchester Royal Infirmary, Oxford Road, Manchester M13 9WL

Objective-To determine the effect of a standard one unit blood donation on stroke distance, blood pressure, and pulse rate in the elderly.

Design-Case-control study.

Setting-Predonation clinic of an autologous blood transfusion service for patients undergoing elective orthopaedic surgery.

Methods-Stroke distance was measured noninvasively in 17 patients using a $2 \mathrm{MHz}$ suprasternal ultrasound probe employing the Doppler principle. Readings were taken over a $10 \mathrm{~min}$ predonation period and compared with readings taken over a 20 min period postdonation. Pulse rate and blood pressure were also recorded at the same time intervals. The patients acted as their own controls. Results-While there was no significant change in pulse and blood pressure in response to a one unit blood donation, stroke distance fell significantly $(\mathrm{P}<0.05)$.

Conclusions-Stroke distance changes earlier in response to blood loss than either pulse rate or blood pressure in the elderly and therefore might be useful in diagnosing hypovolaemia in the early compensated phase.

\begin{tabular}{lllll}
\hline & Pre-analgesia & Post-analgesia & Pvalue & Post-reduction \\
\hline IAL $^{\star}$ & 7.86 & 5.23 & $<0.05$ & 1.67 \\
Entonox & 7.77 & 2.87 & $<0.001$ & 1.40 \\
P value & NS & $<0.05$ & & NS \\
\hline
\end{tabular}

^IAL, intra-articular lignocaine. 
Indications for thoracolumbar radiography in blunt trauma

F M Saunders, I C Phair

The Emergency Department, North Staffordshire General Hospital, Stoke-on-Trent

ATLS states that (a) thoracolumbar spine $x$ rays should be obtained for any patient suspected of sustaining multiple trauma, (b) the finding of a cervical spine injury mandates radiological examination of the entire spinal column. Several North American publications suggest that routine thoracolumbar radiography is not indicated in blunt trauma patients without back pain or tenderness, provided they have no cognitive deficit or painful distracting injury.

Objective-To examine whether these ATLS guidelines were followed in the emergency department of a large district general hospital. Design-Retrospective review of case notes. Subjects-All patients admitted during a two year period with an ISS $\geq 9$ and a diagnosis of cervical, thoracic, or lumbar vertebral fracture.

Results-Of 104 eligible patients, 72 had the required information fully documented. The commonest mechanism of injury was RTA ( $\mathrm{n}$ $=43)$, followed by falls $(n=25)$, contact sports $(n=2)$, and crush injuries $(n=2)$. There were 36 cervical spine fractures $(50 \%)$, 23 thoracic spine fractures (32\%), and 20 lumbar spine fractures $(28 \%)$. Eight patients had fractures in more than one region of their spine. Neurological deficit was present in $38 \%$ of patients with cervical injury, $22 \%$ with thoracic injury, and $10 \%$ with lumbar injury. Of patients sustaining a cervical fracture, $30 \%$ had their thoracolumbar spine imaged, although of those with GCS $<15100 \%$ were imaged. Four patients with clearly documented absence of back pain or tenderness and GCS < 15 had thoracolumbar vertebral fractures; all had concomitant painful distracting injuries.

Conclusions-During the investigation period more selective clinical indications for thoracolumbar radiography were being used than those recommended in ATLS. No patient had their diagnosis missed or delayed by using clinical indications for such imaging.

Epilepsy in the accident and emergency department - developing a code of safe practice

J Ryan, J Lyndon, S Nash

Accident and Emergency Department, Royal

Sussex County Hospital, Eastern Road, Brighton $B N 2$ 5BE

Objectives-To audit the case notes of 1200 patients who have presented to an A\&E department following an epileptic seizure and based on the findings to produce a proforma which (1) highlights common areas of deficiency in the management of such patients and (2) offers guidelines for the management of these patients.

Methods-We audited 1200 case notes of patients who attended an A\&E department following a seizure. 12 hospitals took part in the audit. Each contributed 100 sets of notes. Aspects of care examined included documentation, prehospital care, examination, investigations, treatment, and disposal.

Results-There were large variations in practice between departments for all aspects of care that were analysed. There were a number of important areas where documentation was deficient. The results have cost implications as well as implications for the quality of care offered to patients presenting following an epileptic seizure.

Conclusions-There is great variation between hospitals in the management of patients presenting to an $A \& E$ department following an epileptic fit. We propose the use of a proforma for documentation in the management of patients who present with an epileptic seizure which also includes guidelines on how to manage these patients and offer them optimal care.

Anaesthesia for the reduction of fractures of the distal radius in Scotland

C A Graham, A J Gibson, C M Goutcher, D Scollon ${ }^{\star}$

Department of Orthopaedic Victoria Infirmary, Glasgow; ${ }^{\star}$ Department of Accident and Emergency Medicine, Royal Infirmary, Glasgow

Recent studies have shown the increasing popularity of haematoma blocks throughout the UK. General anaesthesia (GA) remains the commonest method of anaesthesia for fracture reduction and the use of intravenous regional anaesthesia (Bier's block) in the UK is not increasing.

Aims - To determine the methods of anaesthesia used in the immediate management of fractures of the distal radius in adults in Scotland; to compare the Scottish situation with the UK; to assess the cost implications of different anaesthetic and management strategies in the management of these injuries.

Methods-Telephone questionnaire of A\&E doctors in A\&E departments in Scotland which deal with trauma and see more than 20000 patients per annum.

Results - 25 hospitals fulfilled the criteria; all were contacted: $32 \%$ of hospitals (8/25) still use GA routinely for the majority of patients; $20 \%$ of hospitals $(5 / 25)$ still admit all patients overnight for GA; $44 \%$ of hospitals $(11 / 25)$ use Bier's blocks routinely; $12 \%$ use intravenous (IV) sedation (3/25) and $12 \%$ use haematoma blocks (3/25); $4 / 11$ hospitals fast patients before Bier's blocks, 7/11 do not. Training remains patchy and generally poor. Costs are difficult to assess due to lack of available data, but GA (especially with overnight stay) is much more expensive than Bier's block.

Conclusions-Bier's block may be the anaesthetic method of choice for the management of distal radius fractures in Scotland both in terms of efficiency and economics. Training needs to be improved and standardised throughout Scotland. Improved methods of costing procedures accurately would allow more meaningful comparisons between different treatments.

The restriction of extensions to licensing hours does not influence the pattern of alcohol and assault related attendances at an inner city $A \& E$ departmen

C A Graham, L S McLeod, D J Steedman

Department of Accident and Emergency Medicine, Royal Infirmary of Edinburgh, Edinburgh

Many out of hours A\&E attenders are affected by alcohol and many are the victims of violent crime. Extensions to licensing hours increases the frequency of alcohol ingestion in $\mathrm{A} \& \mathrm{E}$ attenders.

Aims - To determine the effect of restriction of licensing extensions on profile of alcohol and assault related attendances at Edinburgh's only adult $\mathrm{A} \& \mathrm{E}$ department.

Methods-Data collection on all patients presenting between $1700 \mathrm{~h}$ and $0900 \mathrm{~h}$ during three two-week periods, immediately before and after restrictions introduced and five weeks after restrictions started. Data collected included age, sex, date and time of presentation, mode of arrival, location of alcohol consumption before attendance, principal diagnosis, and breath alcohol concentration (BAC). Results-5023 patients were seen over six weeks of study. 2187 (43.5\%) did not provide a BAC sample (284 could not or would not give sample, 1903 did not have sample taken for other reasons). Of 2836 patients who gave BAC reading, $71.1 \%(2017 / 2836)$ had a zero reading, $7.8 \%(222 / 2836)$ were positive below legal driving limit $(0.35 \mathrm{mg} / \mathrm{l}), 21.1 \%$ ( $597 /$ 2836) were positive above legal driving limit; $31 \%(253 / 819)$ were admitted as inpatients. 444 patients $(8.8 \%)$ were involved in assaults, 133 were involved with weapons. No significant changes in pattern of attendances following licensing restrictions.

Conclusions-Alcohol and assault related demands on A\&E departments out of hours is high. Changes in licensing laws did not appreciably influence profile of alcohol and assault related attendances at $A \& E$ in this study.

\section{Prehospital rapid sequence induction}

C A Graham, A D McR Meyer

Department of Accident and Emergency Medicine, Royal Infirmary of Edinburgh, Edinburgh

Aims-To determine the number of and reasons for rapid sequence inductions performed by $A \& E$ doctors out of hospital as part of the activities of the MEDIC 1 Flying Squad. Rapid sequence induction was defined in this context as any attempted endotracheal intubation accompanied by the use of drugs to assist intubation and ventilation, including opiates, benzodiazepines, intravenous and topical anaesthetics, and neuromuscular blocking drugs.

Methods-Retrospective case note review of all MEDIC 1 and A\&E records over the period 1 February 1993 to 28 February 1996 (37 months). The anaesthetic technique used, drugs used, complications, difficulties, reasons for induction out of hospital, and grade of doctor performing the technique were determined.

Results-26 patients were treated over 37 months; data for 25 were available-20\% (5/25) had RSI for medical indications: asthma/epilepsy/OD/LVF $\times 2 ; 80 \%(20 / 25)$ had RSI for trauma indications: inadequate airway $70 \%(14 / 20)$, inadequate ventilation $85 \%(17 / 20)$, head injury $85 \%(17 / 20)$, chest injuries $25 \%(5 / 20)$. A variety of anaesthetic techniques was used. No patient acutely deteriorated as a result of prehospital rapid sequence induction.

Conclusions-This is a safe and valuable technique for trained A\&E doctors. Precise benefits of prehospital rapid sequence induction remain to be quantified. 
Information technology in accident and emergency departments R Birkinshaw, J O'Donnell, I Sammy Department of Emergency Medicine, Hope Hospital, Stott Lane, Salford M6 8HD

Objective-To assess how much information technology is available in $A \& E$ departments and how it is used.

Methods-A postal questionnaire was sent to 217 A\&E departments in Great Britain. Responses were obtained from 159 (73.3\%). Results-129 departments (81.1\%) were computerised. Computer data were used for administration in $96.9 \%$, for audit in $79.1 \%$, and for research in only $41.1 \%$. Although most departments had a fax machine, only the minority had the Internet, e-mail, or publication database available. Most departments used several sources of toxicology information, but telephone advice from the Poisons Information Centre was the main source. $74.2 \%$ of hospitals offered courses in computer technology to staff.

Conclusions-There appears to be a variable amount of information technology available and this is not dependent on department size. The technology available is generally underused. We need to attend appropriate courses to get the most from technology available and not get left behind. We display some of the potential facilities available and outline how to get "online."

Quantitative analysis of clinical record audit in the A\&E department and use of a weighted scoring system

C J Mann

Department of Accident and Emergency, Derriford, Plymouth PL6 8DH

Aims-To develop an analytical method to assess the quality of clinical records in A\&E using a simple spreadsheet; to allow numerical performance scores to be derived for each variable, SHO, and clinical record; to standardise the system to enable valid comparisons of doctors and departments over time.

Methods-Each clinical record was assessed by an independent observer. Standard clinical record audit variables, eg, adequacy of history, examination etc, were evaluated. A score of was awarded if the variable was satisfactorily completed, and a score of 0 if the variable was incomplete. 100 records for each SHO were examined: 50 from patients from triage category 1 and 50 from triage category 2 or 3 . Using a standard spreadsheet programme, scores were derived for each record, $\mathrm{SHO}$, and variable. Further refinement of the system was achieved using a weighting system. Each variable was given a weighting factor to reflect it importance for administrative, clinical, and medicolegal purposes.

Results - The results will be demonstrated in table and graphical form

Conclusions-This is a simple system that allows detailed, objective, and reproducible evaluation of clinical records in $\mathrm{A} \& \mathrm{E}$.

Coping with an epidemic of ice injuries: a comparison of two management strategies G Little, D Brooke, R Brown, J Ryan Accident and Emergency Department, Kings College Hospital, Denmark Hill, London SE5 9RS

Objective-To document the experience of two A\&E departments in dealing with a "mini- major incident" brought about by icy weather conditions and compare the different responses of each department to the increased workload.

Methods-A retrospective analysis of all the notes of patients attending the two departments on Saturday 30 December 1995 following a fall on ice. The incident was quantified by comparing the number of attendances on the study day with the number of attendances on the four previous and four subsequent Saturdays. The numbers of calls made to each local ambulance service over a similar period were also studied. The responses made by each department are compared.

Main outcome measures - Numbers of patients, clinical features of those patients who had fallen on ice, the responses of each department to the increased workload.

Results-In department A there was a $45.6 \%$ increase in the number of attendances on the study day, with the peak in the number of hourly attendances between 11:00 h and 15:00 h. Department B saw an increase in attendances of $99.9 \%$, with the peak in attendances between 10:00 $\mathrm{h}$ and 18:00 $\mathrm{h}$ Department A saw 104 patients who attended following a fall on ice, of whom $35.5 \%$ had sustained a fracture; department B saw 177 patients, $44.6 \%$ of those sustaining a fracture Department A dealt with the incident without involving other disciplines, in contrast to department B where other specialties wer called upon to help manage the incident.

Conclusions - The public were inadequately prepared for such adverse weather conditions, despite epidemics of this nature occurring on a regular basis. An epidemic of ice injuries can have significant ramifications with regards to resource allocation for several days after the incident. The two departments each used a different strategy to handle the incident resulting in different resource needs.

Radionuclide bone scanning in the evaluation of suspected scaphoid fracture in an accident and emergency review clinic

C V Egleston, G Campbell-Hewson, S M Robinson, C Maimaris

Accident and Emergency Department, Addenbrooke's Hospital, Hills Road, Cambridge CB2 $2 Q Q$

Objective-To determine the appropriatenes of radionuclide bone scanning in establishing an accurate diagnosis in patients with symp toms and signs suggestive of scaphoid injury attending an A\&E review clinic.

Methods-A retrospective study was conducted using $A \& E$ and radiology records to determine those patients who were booked into the review clinic over a one year period with a suspected scaphoid fracture. The details of all patients who were booked into the clinic were studied. Radiology records were consulted to obtain the results of initia scaphoid radiographs, as well as second radiographs and bone scans where applicable.

Results-162 patients were booked into the $\mathrm{A} \& \mathrm{E}$ review clinic, of whom 128 actually attended. 59 of these attenders were deemed on the basis of clinical grounds and normal repeat radiology to require a radionuclide bone scan to establish an accurate diagnosis of their injury. In 40 cases there was no evidence of fracture (25 scans normal, 11 indicated a soft tissue injury, four indicated the presence of degenerative joint changes). In 19 cases the bone scan indicated the presence of a fracture ( 7 scaphoid, 5 distal radius, 3 trapezium, 3 tri- quetral, 1 capitate). 93 patients had second scaphoid radiographs performed in the clinic of these only two were deemed to show scaphoid fractures that were not apparent on the initial films.

Conclusions - The repeat radiograph at 10-14 $\mathrm{d}$ is an insensitive test for detecting scaphoid fractures. Conversely radionuclide scanning in conjunction with repeat clinical examination had a $32 \%$ fracture pick up rate. We conclude that suspected scaphoid injuries can be effectively pursued to a diagnostic conclusion in A\&E clinics where there is access to radionuclide bone scanning.

Clinical predictors of the circulatory status of an injured adult

F E Lecky

North Western Injury Research Centre and

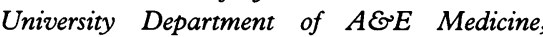
University of Manchester, Oxford Road, Manchester

Objective-To test the hypothesis that other simple bedside tests ("new tests") may more accurately predict the circulatory status of recently injured adults than pulse rate (PR) or blood pressure (BP) ("current tests").

Setting-Emergency department, Hope Hospital, Salford and the trauma unit, Groote Schour Hospital, Observatory, Cape Town.

Methods-A cohort of 239 injured adults was recruited prospectively, $50 \%$ from each of the above settings. All subjects had sustained injuries within the previous $24 \mathrm{~h}$ proximal to the tarsus or carpus which were severe enough to warrant inpatient treatment. The "new tests" for assessing the circulation were: bedside measurements of blood glucose (BG), haemoglobin ( $\mathrm{Hb}$ ), Glasgow coma score (GCS), appearance (pale, sweaty, restless, agitated), pulse oximetry signal, respiratory rate, shock index (SI), stroke distance, and tympanic temperature. These "new" and the "curren tests" were measured in a rapid sequence in each subject before taking a blood sample from an uninjured limb for measurement of plasma lactate and alcohol concentrations.

Outcome measures-Each test's ability to predict circulatory impairment, defined by plasma lactate $\geq 2.5 \mathrm{mmol} / \mathrm{l}$, was analysed through receiver operator curves (ROC), and using McNemar's test for comparing proportions in paired data; comparisons of sensitivity for detecting circulatory impairment at fixed specificities $(74 \%$ and $90 \%)$ and comparisons of specificity for excluding circulatory impairment at fixed sensitivities (61\% and $90 \%$ )

Results-80 patients (34\%) had circulatory impairment as defined by plasma lactate. 78 patients had ISS scores of 16 and over. PR was better than BP (mean, systolic, and diastolic) for predicting circulatory status. ROC curves suggested that GCS, Hb, BG, and SI were generally more accurate predictors than $\mathrm{PR}$ though McNemar's test showed that only GCS and $\mathrm{Hb}$ were significantly $(\mathrm{P}<0.05)$ better at comparable sensitivities/specificities. Conclusions - The data support the hypothesis and suggest that it may be of benefit in initial assessment areas to regularly monitor GCS and $\mathrm{Hb}$ as well as PR and $\mathrm{BP}$ as indicators of the circulatory status in moderately to severely injured adults. 
Human neutrophil elastase release during cardiac arrest

J P Wyatt, C E Robertson

Accident and Emergency Department, Royal Infirmary of Edinburgh, Lauriston Place, Edinburgh EH3 $9 Y W$

Degranulation of neutrophils (thus releasing elastase into the circulation) after a variety of stresses has been implicated in the pathogenesis of certain illnesses, most notably adul respiratory distress syndrome (ARDS) after trauma. Recent work has shown that the release of elastase from neutrophils occurs within minutes of injury, but that the amoun released varies enormously between individuals. The rapid mechanism causing elastase release and the reason for variation in the amount released is unclear. Using cardiac arrest as a model we investigated this phenomenon.

Methods - 51 patients presenting to one A\&E department following out of hospital cardia arrest had blood samples drawn on arrival at hospital. The timing and treatment of cardiac arrest before obtaining the samples was recorded. In particular, the administration of drugs (which depended on the training of the ambulance personnel involved) was carefully documented. After separation by centrifuge plasma was aspirated, frozen, and stored a $-80^{\circ} \mathrm{C}$. Plasma neutrophil elastase was measured in these samples and others obtained from healthy volunteers by specific radioimmunuassay with rabbit polyclonal antiserum. Results-Plasma neutrophil elastase levels were higher $(\mathrm{P}<0.05)$ when analysed using the Mann Whitney $U$ test (non-parametric data) in patients presenting in cardiac arrest (median $75.0 \mathrm{ng} / \mathrm{ml}$ ) than in healthy controls (median $18.6 \mathrm{ng} / \mathrm{ml}$ ). When analysing possible triggers for the release of elastase, the 33 patients treated with intravenous adrenaline before sampling had higher $(P<0.05)$ elastase levels (median $103.0 \mathrm{mg} / \mathrm{ml}$ ) than the 18 who had not received adrenaline (median 52.3 $\mathrm{mg} / \mathrm{ml}$ ).

Conclusions-Cardiac arrest is a stimulus for neutrophils to release elastase into the circula tion. The results suggest that circulating adrenaline may be the trigger responsible for this. If this proved to also be the trigger in ARDS, the implications would be considerable: it might be possible to prevent its development in patients after trauma by the use of specific adrenergic blocking agents.

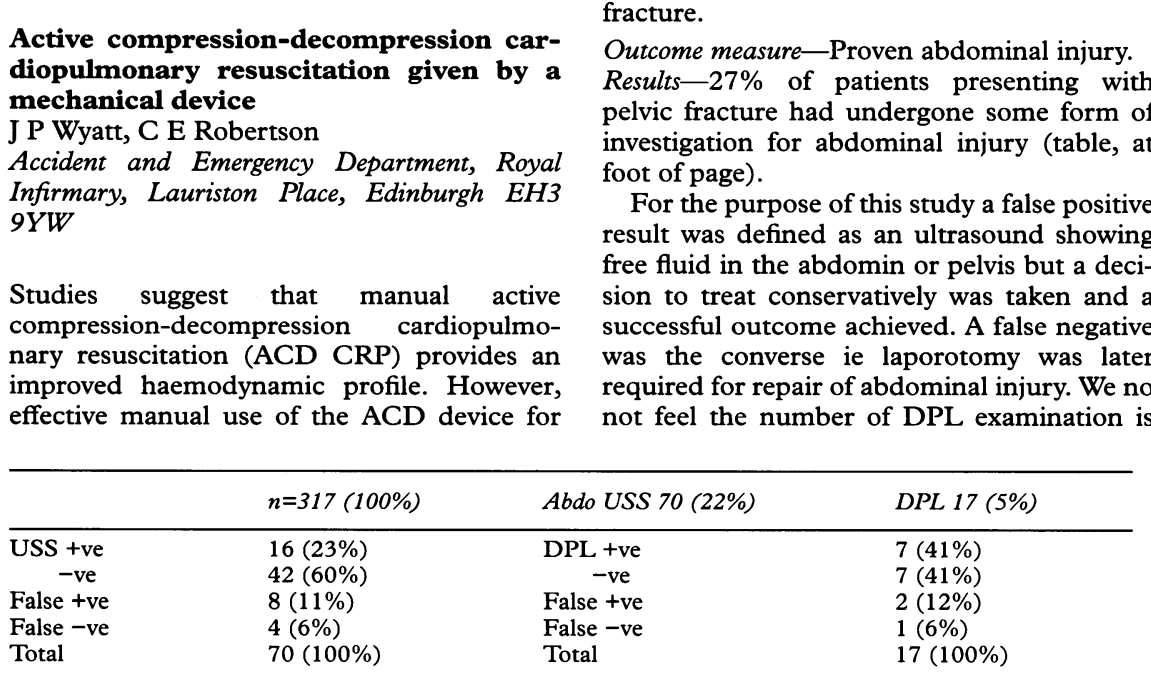

more than a short period is limited by the effort required by an individual to use it. In order to address this problem, a mechanical ACD device has been produced.

Methods-27 adult patients presenting in nontraumatic cardiac arrest to one A\&E department were enrolled into the study. The European Resuscitation Council guidelines for the management of cardiac arrest were followed. Sequential $3 \mathrm{~min}$ periods of standard CPR given by a mechanical "Thumper" were compared with those given by the mechanica ACD device. The following recordings were made: end tidal $\mathrm{CO}_{2}$ (continuous sampling from endotracheal tube), arterial pressures (femoral arterial lines provided continuous traces), arterial blood gases and mixed venous blood gases (from an internal jugular central line). The timing of any return of spontaneous circulation (ROSC) during the study was noted.

Results-Of the five patients who had a period of ROSC, four experienced this during ACD CPR. Similar femoral arterial pressure tracings were obtained during both $\mathrm{ACD}$ and standard CPR. Mean end tidal $\mathrm{CO}_{2}$ during ACD CPR was $8.9 \mathrm{~mm} \mathrm{Hg}$, compared with $7.3 \mathrm{~mm} \mathrm{Hg}$ and $6.9 \mathrm{~mm} \mathrm{Hg}$ during first and second periods of standard CPR respectively, but the differences were not significant when analysed using the Student paired $t$ test. Similarly, there was no significant rise in mixed venous $\mathrm{H}^{+}$or $\mathrm{Pco}_{2}$ during $\mathrm{ACD} \mathrm{CPR}$, when compared with standard CPR.

Conclusions - The results fail to provide evidence that mechanical ACD CPR is more effective haemodynamically than standard mechanical CPR. Considering the result of this study in conjunction with those from recent clinical trials of manual ACD CPR, the future role of $\mathrm{ACD} C P R$ remains in some doubt.

Abdominal injury associated with pelvic fracture: a diagnostic dilemma

M Smith, M V Prescott

North Staffordshire Royal Infirmary, Princess

Road, Newcastle upon Tyne, Stoke on Trent

Design - Retrospective case study.

Setting-North Staffordshire Hospital emergency department.

Subjects-All 317 patients admitted through the emergency department between dates April 1992 and March 1996 with a pelvic fracture.

n abdominal injury. pelvic fracture had undergone some form of

For the purpose of this study a false positive Fas defined as an ultrasound showing sion to treat conservatively was taken and 作 standard three-view series fails to show the cervicothoracic junction, swimmer's views remain useful for visualisation of the vertebra bodies; however, in unconscious patients in whom cervical spine fracture dislocation must be rapidly excluded, right and left supine (trauma) oblique films are more likely to be successful (by demonstration of the posterior elements). They expose patients to less radiation than swimmer's views and are an invaluable additional view in trauma radiology. 
Staff affected by verbal and physical violence in accident and emergency A J Ireland, G W McNaughton

Accident and Emergency Department, Glasgow Royal Infirmary, 84 Castle Street, Glasgow G4 OSF

We set out to determine the number and type of violent incidents occurring in Glasgow Royal Infirmary A\&E department, a busy urban department with 72000 new annual attendances. A prospective questionnaire survey was carried out of all staff for a three month period (February to April 1996). All staff working in A\&E, including ambulance personnel and police in the department, completed a survey form immediately after violent incidents occurred. Staff detailed the type of incident: verbal (with an implicit threat of violence) or physical assault; staff members involved; use of weapons; alcohol or drug intoxication; waiting time and date and times of incidents. 69 questionnaires were completed during the study period. Multiple staff were involved on 37 occasions with 129 staff involved in all (table).

\begin{tabular}{lllll}
\hline & Nurses & Doctors & Others & Total \\
\hline Verbal & 62 & 22 & 14 & 98 \\
Physical & 14 & 14 & 3 & 31 \\
Total & 76 & 36 & 17 & 129 \\
\hline
\end{tabular}

Police were involved in 29 of the 69 incidents reported $(42 \%)$. Of those affected by physical violence ( 31 staff members and one patient), no significant injuries were recorded. Weapons were brandished on four occasions (a knife, a chair, a stick, and a crutch). The majority of offenders were male patients $(75 \%)$ whose average age was 33 (range 18-65). Alcohol intoxication was clinically evident in $76 \%$. Incidents appeared to bear no relation to waiting time or day of the week but $65 \%$ occurred between $2100 \mathrm{~h}$ and $0700 \mathrm{~h}$.

Conclusions-Violence in this A\&E department remains a significant problem for nursing and medical staff. Although there were no significant injuries there was a heavy reliance on a rapid response from the police. The findings of this survey, along with unsolicited local media publicity, have prompted the hospital trust management to undertake an urgent review of security arrangements and department layout in order to try to reduce the number of violent incidents.

\section{Trauma triage: a comparison of CRAMS and TRTS in a United Kingdom popula- tion}

A Gray, E C Goyder, S W Goodacre,

G S Johnson

Accident and Emergency Department, St fames's University Hospital, Beckett Street, Leeds LS9 $7 T F$

The CRAMS and triage version of the revised trauma score (TRTS) are methods of prehospital trauma triage developed to identify patients who may benefit from transportation to a trauma centre. Despite the absence of a formal trauma centre network in the UK these scales are of potential value to seriously injured patients by allowing prearrival activation of hospital trauma teams. We evaluated their use in a sample of the UK trauma population.

Methods-Trauma patients admitted to Leeds General Infirmary A\&E resuscitation room between 1.1 .93 and 7.6.95 who fulfilled MTOS (UK) entrance criteria were identified. Major trauma was defined as an injury severity score of $>15$, admission to an intensive care unit, or hospital death. Patients with incomplete data and transfers from other hospitals were excluded. The ability of each scale to identify major trauma was assessed by calculating the sensitivity and specificity of every point on both scales. These values were plotted on a receiver operator characteristic curve (ROC) to allow comparison. The best cut off value for each scale was defined as the point at which the ratio of sensitivity to specificity was nearest to 1 .

Results-Data were collected on 213 patients, of whom $97(46 \%)$ had major trauma. The best cut off points to identify major trauma were CRAMS $<9$ and TRTS $<12$. At these cut offs the TRTS was significantly more specific but had an inferior sensitivity (NS). Performance of both scales was poor when plotted on the ROC curve.

Conclusions-CRAMS and TRTS were unable to identify major injuries with adequate sensitivity and specificity in our sample to merit their use as a tool to activate trauma teams in the UK. Further research is required to develop a UK specific triage tool or validate existing triage criteria in the UK.

The effect of traumatic brain injury on cardiorespiratory function

G McMahon, E Kirkman ${ }^{\star}$, R Little

North West Injury Research Centre, Stopford Building, University of Manchester, M13 9PT and ${ }^{*}$ Department of Biological Sciences, University of Durham, DH1 3LE

$64 \%$ of trauma deaths have associated traumatic brain injury. The cardiorespiratory consequences of this injury are poorly understood. The aim of this study was to determine the acute cardiovascular, respiratory, and autonomic effects of mild and moderate brain injury. The study was conducted on 15 male Wistar rats $(240-260 \mathrm{~g})$ anaesthetised with alphadolone/alphaxolone. Instantaneous heart rate (HR) was calculated from $R-R$ intervals from the electrocardiogram, mean arterial blood pressure (MBP) through a cannula in the tail artery, and respiratory frequency using a neonatal respiration monitor. R-R interval standard deviation (R-R SD, an index of cardiac parasympathetic activity) was determined only during normal respiration. Normal body temperature was maintained throughout. Three groups of five animals were studied. The first group received a moderate brain injury (1.6-1.8 atm pressure, $10 \mathrm{~ms}$ duration applied directly onto the aura over the parietal lobe through a cannula sealed into the skull); the second group received a mild injury $(1.0-1.2$ atm, $10 \mathrm{~ms})$ the third underwent all of the surgery but not the pressure pulse (control). Measurements were made before, $2 \mathrm{~s}$ after injury, and at 15 $\mathrm{min}$ intervals thereafter for $60 \mathrm{~min}$. Moderate brain injury produced an immediate, significant, fall in HR of 175 (SEM 50) from 465 (7) beats $/ \min (P<0.05$, analysis of variance), a rise in $\mathrm{MBP}$ of 24 (5) from 110 (4) $\mathrm{mm} \mathrm{Hg}$, and apnoea lasting 34 (2) s. Thereafter HR, $\mathrm{MBP}$, and respiration returned to control levels for the remainder of the study. R-R SD was raised significantly $15 \mathrm{~min}$ after moderate brain injury compared to pre-injury: 1.62 (0.16) $v 1.25(0.07) \mathrm{ms}$, respectively, and remained increased for the remainder of the study. Mild injury produced no significant cardiovascular or autonomic changes, but did lead to a brief apnoea of 6 (1)s. Control animals showed no significant changes during the study.

Conclusions-Moderate brain injury produces apnoea, bradycardia, and hypertension, with evidence of increased cardiac parasympathetic tone which outlasts the recorded changes in HR and MBP. These cardiovascular and autonomic changes were not present with mild injury; however, the animals did become apnoeic. This is clinically important because the presence of traumatic brain injury may significantly affect the interpretation of vital signs in the acute multiply injured patient.

Improving the diagnosis of septic olecranon and prepatellar bursitis

I M Stell, G W L Evans

Accident and Emergency Department, Guys Hospital, St Thomas Street, London SE1 9RT

Olecranon and prepatellar bursitis, are relatively common problems presenting to the A\&E department. Clinical features can be helpful in separating septic from non-septic causes, but it is still difficult to be certain in a high proportion of cases. The tests which have been used to help to make this distinction include cell counts on, and culture of, the aspirated fluid. However, false negative culture results on plain samples appear to be common. 27 cases, 19 of olecranon and eight of prepatellar bursitis, were analysed prospectively, with samples of the aspirate being sent where the volume was adequate for each of: cell counts on an automated counter, culture of a plain sample, and inoculation into blood culture bottles. 18 of the 27 cases were septic and $S$ aureus was the infective agent in al cases. The mean white cell count on nonseptic cases was $2.1 \times 10^{9} / 1$, and in septic cases was $12.9 \times 10^{9} / 1$. The difference was statistically significant $(P<0.05)$, but the overlap of the ranges was too great for this to be a useful test. Of the 18 septic cases, 12 had both plain culture, and inoculation into blood cultures performed. Only seven of these 12 had positive cultures on the plain samples, with al 12 of the samples inoculated into blood culture bottles yielding positive cultures (statistically significant difference, $\mathrm{P}<0.02, \chi^{2}$ test).

Conclusions-All cases of olecranon and prepatellar bursitis should be aspirated. The inoculation of this fluid into blood culture bottles will accurately determine whether or not the bursa is infected. This distinction is essential for accurate treatment, to avoid worsening suppuration and sinus formation. 